\title{
HOMOGENEOUS AND NONHOMOGENEOUS DIOPHANTINE EQUATIONS
}

\author{
ANTHONY A. AUCOIN
}

In an earlier paper ${ }^{1}$ we considered homogeneous polynomials $f$ and $g$ whose degrees are relatively prime, and solved the Diophantine equation $f(x)=g(y)$. These results are generalized in the present paper. The solutions are given in terms of arbitrary parameters and if the parameters are integral the solutions are also.

We begin our discussion with the hypothesis that the functions $f(x)=f\left(x_{11}, \cdots, x_{1 m}, \cdots, x_{n 1}, \cdots, x_{n m}\right), g(x)=g\left(x_{11}, \cdots, x_{1 m}, \cdots\right.$, $\left.x_{n 1}, \cdots, x_{n m}\right)$ are polynomials with integral coefficients, homogeneous in each set of variables $x_{k 1} x_{k 2} \cdots x_{k m}$; $f$ being of degree $\alpha_{k} \geqq 0, g$ being of degree $\beta_{k} \geqq 0$ in the sets $x_{k 1} x_{k 2} \cdots x_{k m}$ and $d_{k}=\alpha_{k}-\beta_{k}$. We suppose further that integers $\lambda_{i}, \mu_{i} \geqq 0$ exist such that ${ }^{2}$

$$
\sum_{i=1}^{n} d_{i} \lambda_{i}=-\sum_{i=1}^{n} d_{i} \mu_{i}=1 .
$$

Theorem 1. The Diophantine equation

$$
f(x)=g(x)
$$

has integral solutions, and every solution for which the members of (2) do not vanish, is equivalent (in a sense to be defined) to one of the solutions given by

$$
x_{k j}=\alpha_{k j}[g(\alpha)]^{\lambda_{k}}[f(\alpha)]^{\mu_{k}}, \quad k=1, \cdots, n ; j=1, \cdots, m,
$$

where the $\alpha_{k j}$ are arbitrary integers.

Proof. Let $x_{k j}=\alpha_{k j} s^{\lambda} t^{\mu k}$. Then by (1), (2) becomes $s f(\alpha)=\operatorname{tg}(\alpha)$, which is satisfied identically in the $\alpha_{k j}$ if $s=g(\alpha), t=f(\alpha)$. Hence (3) is a solution of (2).

We now define the concept of equivalent solutions. Suppose $x_{k j}=\rho_{k j}$ is a solution of (2). If there are no integers $b>1, \rho_{k j}^{\prime}$ such that $\rho_{k j}=\rho_{k j} b^{\sigma_{k}}$, where the $\sigma_{k}$ are positive integers such that $\sum_{k=1}^{n} \alpha_{k} \sigma_{k}$

Received by the editors March 2, 1942.

${ }^{1}$ A. A. Aucoin and W. V. Parker, Diophantine equations whose members are homogeneous, this Bulletin, vol. 45 (1939), pp. 330-333.

2 We need to postulate only the existence of $\lambda_{i}$ since it may be shown that for $n$ odd $\mu_{i}=\sum_{j=1}^{n} d_{j}-d_{i}-2 \sum_{k=1}^{(n-1) / 2} d_{i+k}-\lambda_{i}$ where $d_{n+p}=d_{p}$, and for $n$ even $\mu_{i}=\sum_{j=1}^{n} d_{j}-d_{i}$ $-2 \sum_{k=1}^{(n / 2)-1} d_{i+k}-2 d_{n / 2+i}^{\prime}-\lambda_{i}$ where $d_{k}^{\prime}=d_{k}$ for $k \leqq n, d_{k}^{\prime}=0$ for $k>n$ and $d_{n+p}=d_{p}$. We must, however, assume that $\mu_{i} \geqq 0$.

${ }^{3}$ It will be shown later that $s, t \neq 0$. 
$=\sum_{k=1}^{n} \beta_{k} \sigma_{k}$, then $x_{k j}=\rho_{k j}$ is defined to be a primitive solution of (2). If $x_{k j}=\rho_{k j}$ is a primitive solution of (2), then $x_{k j}=\rho_{k j} b^{\sigma_{k}}$ (derived from the primitive solution), where $b$ is a nonzero integer, ${ }^{4} \sigma_{k}$ are positive integers such that $\sum_{k=1}^{n} \alpha_{k} \sigma_{k}=\sum_{k=1}^{n} \beta_{k} \sigma_{k}$, is also a solution. Two solutions are said to be equivalent if they may be derived from the same primitive solution.

Suppose now that $x_{k j}=\rho_{k j}$ is a solution of (2). Then $f(\rho)=g(\rho)$. If we choose $\alpha_{k j}=\rho_{k j}$, (3) becomes $x_{k j}=\rho_{k j}[f(\rho)]^{\lambda_{k}+\mu_{k}}$ which is equivalent to the given solution $x_{k j}=\rho_{k j}$ provided $f(\rho) \neq 0$, since from (1), $\sum_{k=1}^{n} \alpha_{k}\left(\lambda_{k}+\mu_{k}\right)=\sum_{k=1}^{n} \beta_{k}\left(\lambda_{k}+\mu_{k}\right)$. If $f(\rho) \neq 0$, then $s, t \neq 0$.

As our next topic we suppose that for the functions considered above, $f$ is of degree $-\alpha_{p}$ and $g$ is of degree $-\beta_{p}$ in the set $x_{p 1} x_{p 2} \ldots$ $x_{p m}$, where $\alpha_{p}, \beta_{p}$ are positive integers. ${ }^{5}$ Here we let $d_{p}=\beta_{p}-\alpha_{p}$.

Theorem 2. The Diophantine equation

$$
f(x)=g(x)
$$

has solutions, and every solution, for which the members of (4) do not vanish, is equivalent to a solution given by

$$
x_{k j}=\alpha_{k j}[A(\alpha) B(\alpha)]^{\lambda_{k}+\mu_{k}}[g(\alpha)]^{\lambda_{k}}[f(\alpha)]^{\mu_{k}},
$$

where $A(x)=\prod_{j=1}^{m} x_{p_{j}}^{\alpha p}, B(x)=\prod_{j=1}^{m} x_{p j}^{\beta p}$, the $\alpha_{k j}$ being arbitrary integers.

Proof. If we multiply (4) by $A(x) B(x)$ we have

$$
A(x) B(x) f(x)=A(x) B(x) g(x),
$$

each member of which is a polynomial. If we let

$$
x_{k j}=\alpha_{k j} s^{\lambda k} t^{\mu k},
$$

equation (6) becomes ${ }^{6} s A(\alpha) B(\alpha) f(\alpha)=t A(\alpha) B(\alpha) g(\alpha)$, which is identically satisfied in the $\alpha_{k j}$ if $s=A(\alpha) B(\alpha) g(\alpha), t=A(\alpha) B(\alpha) f(\alpha)$. Hence (5) is a solution of (4).

It is evident that more than one of the corresponding sets of variables may be of negative degree.

Suppose that $x_{k j}=\rho_{k j}$ is a given solution of (4). Then $f(\rho)=g(\rho)$. If we choose $\alpha_{k j}=\rho_{k j}$, (5) becomes

$$
x_{k j}=\rho_{k j}[A(\rho) B(\rho) f(\rho)]^{\lambda_{k}+\mu_{k}}
$$

which is equivalent to the given solution $x_{k j}=\rho_{k j}$ provided $f(\rho) \neq 0$, since from (1)

${ }^{4}$ If $b=0$, the solution is trivial.

${ }_{5}^{5}$ The $\lambda_{i}$, and hence the $\mu_{i}$, here are different from those of Theorem 1 .

${ }^{6}$ It will be shown later that $s, t \neq 0$. 


$$
\sum_{k=1}^{n} \alpha_{k}\left(\lambda_{k}+\mu_{k}\right)-2 \alpha_{p}\left(\lambda_{p}+\mu_{p}\right)=\sum_{k=1}^{n} \beta_{k}\left(\lambda_{k}+\mu_{k}\right)-2 \beta_{p}\left(\lambda_{p}+\mu_{p}\right) .
$$

If $f(\rho) \neq 0$ then $s, t \neq 0$. We now pass on to our next result.

Suppose that $f$ is of degree $-\alpha_{p}$ in the set $x_{p 1} x_{p 2} \cdots x_{p m}$ and $g$ is of degree $-\beta_{q}$ in the set $x_{q 1} x_{q 2} \cdots x_{q m}$ where $\alpha_{p}, \beta_{q}$ are positive integers. Here ${ }^{7} d_{p}=-\left(\alpha_{p}+\beta_{p}\right), d_{q}=\alpha_{q}+\beta_{q}$.

THEOREM 3. The Diophantine equation $f(x)=g(x)$ has solutions, and every solution for which the members do not vanish, is equivalent to a solution given by $x_{k j}=\alpha_{k j}[C(\alpha) D(\alpha)]^{\lambda_{k}+\mu_{k}}[g(\alpha)]^{\lambda_{k}}[f(\alpha)]^{\mu_{k}}$ where $C(x)$ $=\prod_{j=1}^{m} x_{p j}^{\alpha_{p}}, D(x)=\prod_{j=1}^{m} x_{q j}^{\beta q}$, the $\alpha_{k j}$ being arbitrary integers.

The proof is similar to that of Theorem 2.

We can now extend the above methods of solution to nonhomogeneous equations. The nature of this extension is contained in the following theorem.

TheOREM 4. The Diophantine equation ${ }^{8}$

$$
f(x)=g(x)
$$

where $f(x)=\sum_{h=1}^{m} a_{h} \prod_{i=1}^{n} x_{i}^{\alpha_{h i}}, g(x)=\sum_{k=1}^{p} b_{k} \prod_{j=1}^{q} x_{j}^{\beta_{k j}}, a_{h}, b_{k}$ are integers, $a_{h i}, \beta_{k j}$ positive integers, has solutions if there exist positive integers $u_{i}, v_{j}, M, N$ such that

$$
\begin{array}{ll}
\sum_{i=1}^{n} \alpha_{h i} u_{i}=M+1, & h=1, \cdots, m, \\
\sum_{j=1}^{q} \beta_{k j} u_{j}=M, & k=1, \cdots, p, \\
\sum_{i=1}^{n} \alpha_{h i} v_{i}=N, & h=1, \cdots, m, \\
\sum_{j=1}^{q} \beta_{k j} v_{j}=N+1, & k=1, \cdots, p,
\end{array}
$$

and every solution for which the members of (8) do not vanish, is equivalent to a solution given by

$$
x_{i}=\alpha_{j}[g(\alpha)]^{u_{i}}[f(\alpha)]^{v_{i}},
$$

where the $\alpha_{j}$ are arbitrary integers.

${ }^{7}$ As in the previous theorem the $\lambda_{i}$ and $\mu_{i}$ will be different from those of Theorem 1 .

${ }^{8}$ A. A. Aucoin and W. V. Parker, op. cit., p. 331. Theorem 4, which generalizes Theorem 2 of the reference may be obtained from that theorem directly by setting $y_{j}=x_{i}$. 


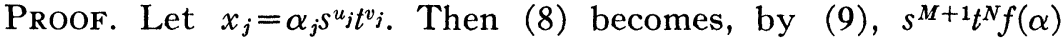
$=s^{M} t^{N+1} g(\alpha)$, which is satisfied identically in the $\alpha_{j}$ if $s=g(\alpha)$, $t=f(\alpha)$. Hence $(10)$ is a solution of (8).

When the conditions of (9) are satisfied there also exist nonnegative integers $R, u_{j}^{\prime}$ such that

$$
\sum_{i=1}^{n} \alpha_{h i} u_{i}^{\prime}=R, \sum_{j=1}^{q} \beta_{k j} u_{j}^{\prime}=R, \quad h=1, \cdots, m ; k=1, \cdots, p .
$$

If $x_{j}=\rho_{j}$ is a solution of (8) and there are no integers $d>1, u_{j}^{\prime}, \rho_{j}^{\prime}$ such that $\rho_{j}=\rho_{j}^{\prime} d^{u_{j}^{\prime}}$ where $u_{j}^{\prime}$ satisfies (11), then $x_{j}=\rho_{j}$ is defined to be a primitive solution of (8). If $x_{j}=\rho_{j}$ is a primitive solution of (8), then $x_{j}=\rho_{j} d_{j}^{u_{j}^{\prime}}$ (derived from the primitive solution) where $d(\neq 0)$ is an integer and $u_{j}^{\prime}$ are non-negative integers satisfying (11), is also a solution.

Suppose $x_{j}=\rho_{j}$ is a solution of (8). Then $f(\rho)=g(\rho)$. If we choose $\alpha_{j}=\rho_{j}$, (10) becomes $x_{j}=\rho_{j}[f(\rho)]^{u_{j}+v_{j}}$ which is equivalent to the solution $x_{j}=\rho_{j}$ since from (9)

$$
\sum_{i=1}^{n} \alpha_{h i}\left(u_{i}+v_{i}\right)=\sum_{j=1}^{q} \beta_{k j}\left(u_{j}+v_{j}\right)=M+N+1
$$

In Theorems 2 and 3 it is conceivable that the method applies to cases other than those in which an entire set of variables appear in the denominator. The cases are too numerous to consider. It is likewise apparent that the method of Theorem 4 applies when some of the exponents are negative. As there is no typical case the procedure will be illustrated by a particular example.

Consider the equation

$$
a x^{3} u^{5} / v^{7}+b y^{4} v^{4} / u^{2}=c x^{5} / w
$$

which may be written as

$$
a x^{3} u^{7} w+b y^{4} v^{11} w=c x^{5} u^{2} v^{7} .
$$

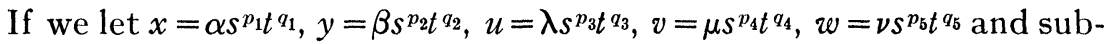
stitute in (13) we have

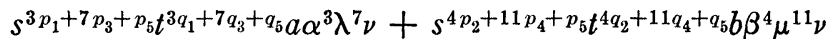

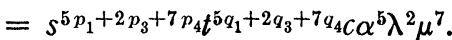

We require an integral solution of the equations

$$
\begin{array}{ll}
3 p_{1}+7 p_{3}+p_{5}=m, & 3 q_{1}+7 q_{3}+q_{5}=n+1, \\
4 p_{2}+11 p_{4}+p_{5}=m, & 4 q_{2}+11 q_{4}+q_{5}=n+1,
\end{array}
$$




$$
5 p_{1}+2 p_{3}+7 p_{4}=m+1, \quad 5 q_{1}+2 q_{3}+7 q_{4}=n .
$$

A solution of these equations is $p_{1}=4, p_{2}=3, p_{3}=11, p_{4}=7, p_{5}=1$, $q_{1}=4, q_{2}=3, q_{3}=11, q_{4}=7, q_{5}=3$. Hence a solution of (12) is $x=\alpha s^{4} t^{4}$, $y=\beta s^{3} t^{3}, \quad u=\lambda s^{11} t^{11}, \quad v=\mu s^{7} t^{7}, \quad w=\nu s t^{3} \quad$ where $s=a \alpha^{3} \lambda^{7} \nu+b \beta^{4} \mu^{11} \nu$, $t=c \alpha^{5} \lambda^{2} \mu^{7}$.

If $x=x^{\prime}, y=y^{\prime}, u=u^{\prime}, v=v^{\prime}, w=w^{\prime}$ is a given solution of (12) and the choice $\alpha=x^{\prime}, \beta=y^{\prime}, \lambda=u^{\prime}, \mu=v^{\prime}, \nu=w^{\prime}$ is made then $s=t$ and the solution becomes $x=x^{\prime} t^{8}, y=y^{\prime} t^{6}, u=u^{\prime} t^{22}, v=v^{\prime} t^{14}, w=w^{\prime} t^{4}$ which is equivalent to the given solution provided $t \neq 0$.

United States Naval Academy and

UNIVERSITY OF HOUSTON

\section{VECTOR ANALOGUES OF MORERA'S THEOREM}

E. F. BECKENBACH

Let the vector

$$
X \equiv X\left(x_{1}, x_{2}, x_{3}\right) \equiv X(x) \equiv X_{1} i+X_{2} j+X_{3} k
$$

be defined and continuous in the domain (non-null connected open set) $D$. Consider the mean-value vector

$$
\boldsymbol{X}^{(\rho)}(x) \equiv \frac{1}{\left|V_{\rho}\right|} \int_{V_{\rho}} \boldsymbol{X}(x+\xi) d V,
$$

where $V_{\rho}$ denotes the sphere

and $\left|V_{\rho}\right|$ its volume,

$$
\xi_{1}^{2}+\xi_{2}^{2}+\xi_{3}^{2}<\rho^{2},
$$

$$
\left|V_{\rho}\right| \equiv 4 \pi \rho^{3} / 3 \text {. }
$$

The vector (1) can be defined thus for only a part $D_{\rho}$ of $D$, but this is of no consequence since $\rho$ is arbitrarily small.

Since $\boldsymbol{X}(x)$ is continuous, it follows that $\boldsymbol{X}^{(\rho)}(x)$ has continuous partial derivatives of the first order; these are given by

$$
\frac{\partial}{\partial x_{p}} \boldsymbol{X}^{(\rho)}(x)=\frac{1}{\left|V_{\rho}\right|} \int_{S_{\rho}} \boldsymbol{X}(x+\rho \alpha) \alpha_{p} d \sigma,
$$

where $S_{\rho}$ denotes the surface of $V_{\rho}$ and $\alpha_{1}, \alpha_{2}, \alpha_{3}$ are the components of the unit vector along the outer normal to $S_{\rho}$.

Presented to the Society, September 5, 1941 under the title Vector formulations of Morera's theorem; received by the editors March 3, 1942. 